\title{
A splicing enhancer exhibits both constitutive and regulated activities
}

\author{
Ming Tian' and Tom Maniatis ${ }^{2}$ \\ Department of Biochemistry and Molecular Biology, Harvard University, Cambridge, Massachusetts 02138 USA
}

The Drosophila proteins Transformer (Tra) and Transformer2 (Tra2) regulate the sex-specific alternative splicing of Drosophila doublesex (dsx) pre-mRNA by specifically binding to a splicing enhancer (dsx repeat element; dsxRE) located 300 nucleotides (nt) downstream from a female-specific $3^{\prime}$ splice site. In this paper we show that the $d s x R E$ can function as a Tra and Tra2-independent splicing enhancer in vitro when located within 150 nucleotides of the $3^{\prime}$ 'splice site. Based on the relative levels of SR proteins that bind stably to the $d s x R E$ in the presence or absence of Tra and Tra2, we propose that the constitutive splicing activity of the $d s \times R E$ is mediated by its weak interactions with SR proteins and possibly other general splicing factors. In contrast, Tra and Tra2 allow the $d s x R E$ to function at a distance from the intron by stabilizing the interactions between these proteins and the $d s \times R E$.

[Key Words: Splicing enhancer; positive regulation; pyrimidine tract]

Received March 16, 1994; revised version accepted May 20, 1994.

The Drosophila proteins Transformer (Tra) and Transformer2 (Tra2) mediate the positive regulation of doublesex $(d s x)$ female-specific pre-mRNA splicing by binding to a regulatory element located in the exon downstream of a female-specific $3^{\prime}$ splice site (Nagoshi and Baker 1990; Hedley and Maniatis 1991; Hoshijima et al. 1991; Ryner and Baker 1991; Tian and Maniatis 1992; 1993). This regulatory sequence, which is characterized by six copies of a 13-nucleotide repeat sequence, is called the $d s \times$ repeat element ( $d s \times R E)$. The $d s x R E$ has been shown to be both necessary and sufficient for Tra and Tra2-dependent, female-specific splicing of $d s x$ premRNA in vivo (Hedley and Maniatis 1991; Hoshijima et al. 1991; Ryner and Baker 1991) and in vitro (Tian and Maniatis 1992). In addition, the $d s x R E$ is capable of suppressing both branchpoint sequence and pyrimidine tract mutations in heterologous introns (Tian and Maniatis 1992). Thus, the $d s x$ RE functions as a Tra and Tra2-dependent splicing enhancer.

Both Tra (Boggs et al. 1987) and Tra2 (Amrein et al. 1988; Goralski et al. 1989) contain arginine/serine-rich (RS) domains, which are also found in the SR family of general splicing factors (Ge et al. 1991; Krainer et al. 1991; Fu and Maniatis 1992; Zahler et al. 1992), the splicing factor U2AF (Zamore et al. 1992), the U1 small nuclear ribonucleoprotein (snRNP) 70K protein (Theissen et al. 1986; Mancebo et al. 1990), and the negative regulator of alternative splicing suppressor of white apri$\cot \left[s u\left(w^{a}\right)\right]$ (Chou et al. 1987). Recent studies have

\footnotetext{
${ }^{1}$ Present address: Howard Hughes Medical Institute, The Children's Hospital, Boston, Massachusetts 02115 USA.

${ }^{2}$ Corresponding author.
}

shown that RS domains mediate associations between proteins containing this motif (Wu and Maniatis 1993; Amrein et al. 1994; Kohtz et al. 1994) and are responsible for directing splicing factors to subnuclear regions (speckles) where many splicing factors are concentrated (Fu and Maniatis 1990; Li and Bingham 1991; Spector et al. 1991; M.L. Hedley, H. Amrein and T. Maniatis, in prep.). Tra2 also has an RNA recognition motif (RRM) that mediates protein-RNA interactions (for review, see Bandziulis et al. 1989; Kenan et al. 1991). In contrast, Tra does not contain an RRM. Both the RS domain and the RRM are essential for the in vivo functions of Tra2 (Amrein et al. 1994).

Although the $d s \times R E$ can function as a regulated splicing enhancer, another type of splicing enhancer /the purine-rich element) can function constitutively (Lavigueur et al. 1993; Sun et al. 1993a,b; Watakabe et al. 1993; Xu et al. 1993; Dirksen et al. 1994; Tanaka et al. 1994). Although distinct from each other in sequence, the $d s x R E$ and the purine-rich elements share several properties. Both types of elements are located in the exon and activate the splicing of upstream introns. In addition, both the $d s x R E$ and the purine-rich elements can mediate the activation of heterologous splice sites. Finally, both elements serve as binding sites for splicing factors. Tra and Tra2 bind cooperatively with members of the SR family of general splicing factors to the $d s \times R E$ and promote the formation of a splicing enhancer complex (Tian and Maniatis 1993). SR proteins and U1 snRNA have been shown to interact with purine-rich elements (Lavigueur et al. 1993; Sun et al. 1993b; Watakabe et al. 1993).

The primary difference between these two types of 
splicing enhancers is that the $d s x \mathrm{RE}$ requires Tra and Tra2, whereas the activity of the purine-rich element is constitutive. An additional difference is the relative positions of these elements with respect to the regulated introns. The $d s x R E$ is separated from the female-specific $3^{\prime}$ splice site by 300 nucleotides, whereas the purine-rich elements are generally located closer to the regulated intron. A recent study showed that a purine-rich element in the human fibronectin alternate ED1 exon loses its activity when placed beyond 293 nucleotides downstream of the 3' splice site (Lavigueur et al. 1993).

In this study we have investigated the characteristics of the $d s x R E$ and its relationship to the purine-rich splicing enhancers. We find that the activity of the $d s x \mathrm{RE}$ is a function of its distance from the $3^{\prime}$ splice site. When the element is located at a distance from the $3^{\prime}$ splice site, its activity depends on Tra and Tra2. When placed close to the $3^{\prime}$ splice site the $d s x \mathrm{RE}$ can function as a constitutive splicing enhancer, analogous to the purinerich elements. The constitutive activity of the $d s x \mathrm{RE}$ may be mediated by SR proteins that can bind weakly to the $d s \times R E$ in the absence of Tra and Tra2.

\section{Results}

\section{Tra and Tra2-independent activity of the $\mathrm{dsx} R E$}

To determine whether the activity of the $d s x \mathrm{RE}$ is affected by its distance from the female-specific $3^{\prime}$ splice site, we analyzed the effects of altering this distance on $d s \mathrm{x}$ female-specific splicing in HeLa cell nuclear extracts. As shown previously, activation of the femalespecific 3' splice site requires Tra and Tra2 when the $d s \times \mathrm{RE}$ is located in its original position, 300 nucleotides downstream of the $3^{\prime}$ splice site (Tian and Maniatis 1992; Fig. 1A, D1, lanes 1 and 2). Reducing the length of the spacer sequence to 190 nucleotides appeared to destabilize the pre-mRNA in nuclear extracts, but femalespecific splicing remained Tra and Tra2-dependent (Fig. 1A, D2, lanes 3 and 4). Remarkably, when the length of the spacer sequence was reduced to 150 nucleotides, a significant level of splicing was observed in the absence of Tra and Tra2 (Fig. 1A, D3, lane 5). Addition of Tra and Tra2 appeared to stabilize the RNA but did not significantly change the ratio of splicing product to unspliced precursor (Fig. IA, D3, lane 6). Further truncation of the spacer sequence to 100 nucleotides resulted in efficient female-specific splicing in the absence of Tra and Tra2, and the addition of Tra and Tra2 had no effect (Fig. 1A, D4, lanes 7 and 8). The constitutive female-specific splicing observed with D3 and D4 was dependent on the repeat sequence because female-specific splicing was not observed in the absence of the $d s \times R E$ [Tian and Maniatis 1992; see also Fig. 3A, D16, lane 1 (below)]. These results show that the $d s \times R E$ can function as a constitutive splicing enhancer when located near the female-specific $3^{\prime}$ splice site. An alternative possibility is that the deleted spacer sequence normally blocks the constitutive activity of the $d s \times \mathrm{RE}$, and Tra and Tra2 are required to overcome this block. This possibility was ruled out by show- ing that $d s \times R E$ remained Tra and Tra2-dependent when the normal spacer sequence was substituted by a number of different sequences of the same size (data not shown).

Compared with the Tra and Tra2-dependent regulation, the constitutive activity of the $d s \times \mathrm{RE}$ is somewhat more efficient in activating the female-specific $3^{\prime}$ splice site (Fig. 1B, cf. lanes 1-5 with 6-10). To compare the constitutive activities of the $d s \times R E$ with that of a purinerich element, we replaced the $d s x \mathrm{RE}$ with three copies of a purine-rich sequence from the IgM M2 exon (Watakabe et al. 1993/. As measured by the ratio of unspliced precursor to splicing product, the purine-rich element appeared to activate splicing to a similar extent as the $d s x \mathrm{RE}$, although the RNA containing the purine-rich element was less stable during the incubation in nuclear extracts (Fig. 1B, cf. lanes 11-15 with 6-10).

In spite of similar constitutive activities, the $d s \times R E$ is distinguishable from the purine-rich element by Tra and Tra2 regulation, which allows the $d s x \mathrm{RE}$ to function at a distance from the regulated intron. Consistent with published reports, the purine-rich element lost its activity when placed 300 nucleotides downstream of the 3 ' splice site, and Tra and Tra2 had no effect on its function (Fig. 1C, lanes 1,2 ). In contrast, Tra and Tra 2 could efficiently activate the female-specific $3^{\prime}$ splice site when the $d s x$ RE was placed at the same distance from the 3 ' splice site (Fig. 1C, lanes 3,4). To test whether Tra and Tra2 can function at a distance $>300$ nucleotides, we moved the $d s x$ RE farther away from the female-specific 3 ' splice site by inserting a 200-nucleotide fragment. Significantly, Tra and Tra2 could efficiently activate the female-specific $3^{\prime}$ splice site when the $d s \times R E$ was $500 \mathrm{nu}-$ cleotides downstream of the $3^{\prime}$ splice site (Fig. 1C, lanes $5,6)$. Furthermore, the splicing efficiency remained essentially unchanged with the insertion, implying that the Tra and Tra2-dependent regulation may function at even greater distances from the $3^{\prime}$ splice site (Fig. 1C, cf. lanes 5-6 with 3-4).

In addition to analyzing the effect of distance on positive regulation, we also tested whether the $d s x$ RE can function at locations other than downstream of the $3^{\prime}$ splice site. Specifically, we found that the $d s x$ RE failed to function when located either in the 5' exon upstream of the $5^{\prime}$ splice site or within the intron (data not shown).

\section{Suppression of $3^{\prime}$ splice site mutations by the $\mathrm{dsx} R E$}

The weak constitutive activity of the $d s x$ female-specific $3^{\prime}$ splice site is attributable to a nonconsensus pyrimidine tract that is interrupted by purines (Burtis and Baker 1989; Hoshijima et al. 1991). Tra and Tra2 activate the female-specific $3^{\prime}$ splice site by suppressing the adverse effect of purine substitutions in the pyrimidine tract. In this study we show that the constitutive activity of the $d s x R E$ can also activate the female-specific $3^{\prime}$ splice site in the absence of Tra and Tra2, and exhibits somewhat higher activity compared with the Tra and Tra2-dependent regulation. To further compare the constitutive and Tra and Tra2-dependent activities of $d s \times R E$, we tested 
A
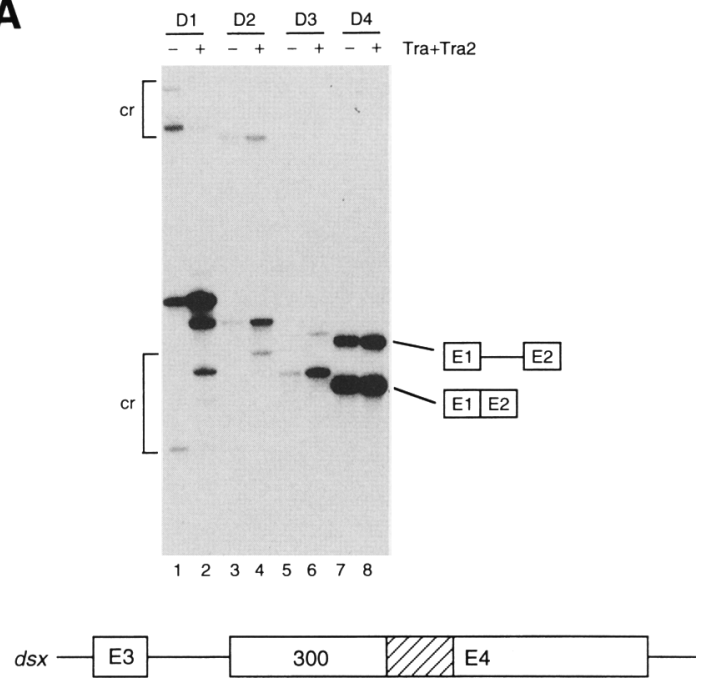

D1

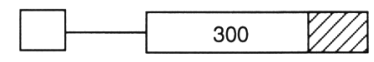

D2

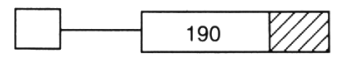

D3

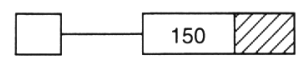

D4

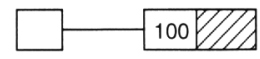

C
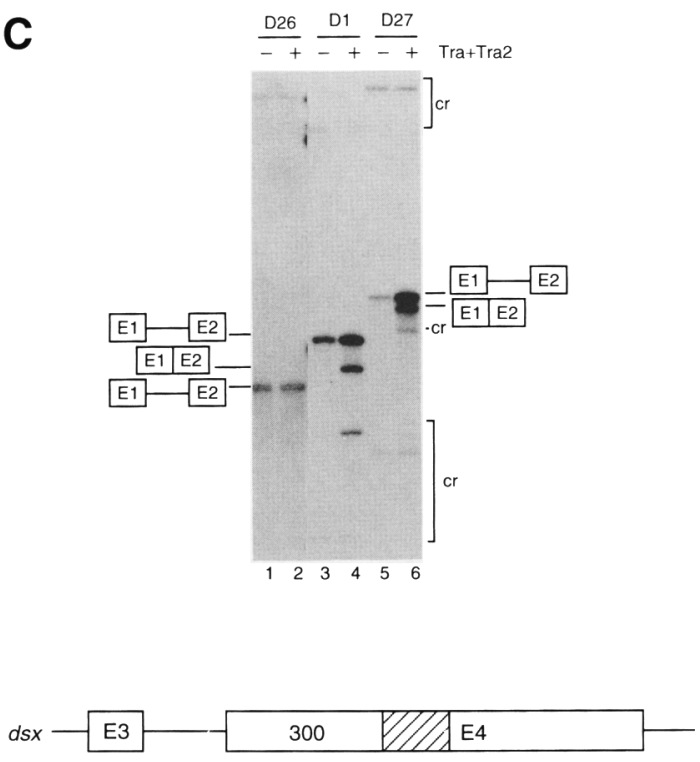

D1

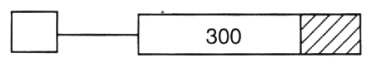

D26

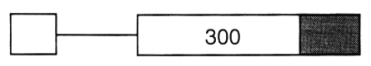

D27

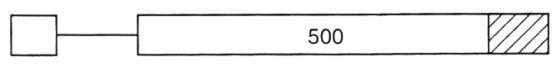

B
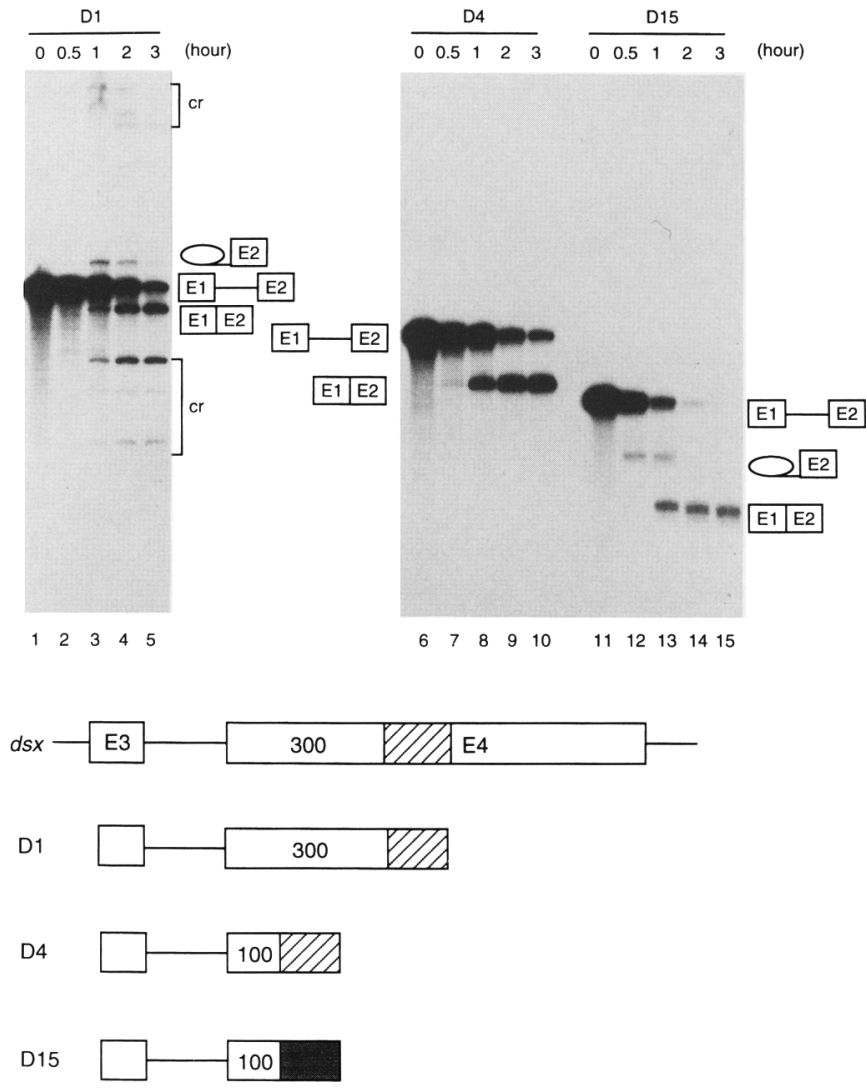

Figure 1. Tra and Tra2-independent activity of the $d s x$ RE. (A) Autoradiograph of denaturing polyacrylamide gel analysis of in vitro splicing reactions using pre-mRNAs in which the distance between the $3^{\prime}$ splice site and the $d s \times R E$ is progressively decreased. The positions of the unspliced pre-mRNAs and ligated exons are diagramed to the right of the autoradiograph. Because of the deletions, the precursors and splicing products decrease in size from the left to right and are labeled at an angle. Because the splicing reactions were incubated for $2.5 \mathrm{hr}$, the amount of splicing intermediates is low and the intermediates are not labeled. (cr) The splicing products involving cryptic splice sites. (Top) - and + indicate the absence or presence of Tra and Tra2 in the splicing reactions, respectively; the pre-mRNAs (D1-D4) used in the reactions are also indicated. The relevant region of $d s x$ pre-mRNA from which the in vitro splicing substrates are derived is diagramed below the autoradiograph and includes the last common exon (E3), the intron, and the female-specific exon (E4). The slashed region in E4 represents the dsxRE. The number in $\mathrm{E} 4$ indicates the number of nucleotides between the femalespecific 3' splice site and the first copy of the repeat sequence. The in vitro splicing substrates (D1-D4) are diagramed below $d s x$. (B) A time course of the in vitro splicing reactions. The autoradiographs are labeled as in $A$. The time of incubation in hours is indicated above each lane. The shaded area in D15 represents the purine-rich element. (C) Analysis of the effects of increasing the distance between the $d s x \mathrm{RE}$ (D27) or the purine-rich element (D26) and the 3' splice site. Because of the instability of D26 RNA, lanes 1 and 2 were exposed five times longer than lanes 3-6. 
their abilities to suppress more extensive purine substitutions in the pyrimidine tract.

The longest stretch of pyrimidines in the nonconsensus pyrimidine tract of $d s x$ female-specific $3^{\prime}$ splice site contains 6 continuous pyrimidines. We disrupted this 6-pyrimidine stretch with 3 purine-substitutions [UCUCUCGAUCUGAUCUAAACCAG/G (wild type) was changed to UCgaUgGAUCUGAUCUAAACCAG/G; the mutations are in lowercase letters]. Significantly, these mutations completely abolished the activation of the female-specific $3^{\prime}$ splice site by Tra and Tra2 when the $d s \times R E$ was located beyond 190 nucleotides downstream of the $3^{\prime}$ splice site (Fig. 2A, D7, D8, lanes 1-4). The small amount of remaining splicing products and intermediates results from splicing events involving cryptic 3 ' splice sites in the female-specific exon. In contrast to the complete inhibition of Tra and Tra2-dependent regulation, these pyrimidine tract mutations had only a mild effect on the constitutive activity of the $d s x$ RE. A significant level of splicing was observed when the $d s x R E$ was placed near the mutant $3^{\prime}$ splice site (Fig. $2 A$, cf. lanes 5 and 8 with 7). The addition of Tra and Tra2 has a small inhibitory effect on splicing, presumably because of steric hindrance caused by the assembly of a large Tra and Tra2-dependent complex near the splice site (Fig. 2A, lane 6). A similar negative effect of Tra and Tra2 on splicing was occasionally observed for D4 as well (data not shown).

Previous studies demonstrated that the first step of the splicing reaction is independent of the $3^{\prime}$ AG in the presence of a strong pyrimidine tract (Reed 1989). Similarly, when the $3^{\prime}$ AG of the female-specific 3' splice site was deleted, efficient accumulation of splicing intermediates, the released $5^{\prime}$ exon and the lariat intermediate, was observed (Fig. 2B, lane 1). We noticed that the $d s \times \mathrm{RE}$ contains a stretch of pyrimidines (UUCUCUUCUCUUC). It was therefore possible that this sequence could function as a pyrimidine tract in the first step of the splicing reaction. Furthermore, the $d s \times R E$ used in these experiments is 220 nucleotides in length and may contain other unidentified sequence elements responsible for the constitutive activity of $d s x \mathrm{RE}$. To rule out these possibilities, we replaced the repeat-containing fragment with four copies of an oligonucleotide corresponding to the repeat sequence but lacking the surrounding sequences. We found that the synthetic dsxRE functions indistinguishably from the original $d s x \mathrm{RE}$ (Fig. 2B, lanes $2,3)$. Therefore, the repeat sequence is sufficient to mediate the constitutive activity of the $d s x$ RE. Similarly, the purine-rich element can also efficiently suppress the same pyrimidine tract mutations and mediate the first step of splicing when the $3^{\prime}$ AG is deleted (Fig. 2C, lanes $1-3)$. These results show that the constitutive activities of the $d s \times \mathrm{RE}$ and the purine-rich elements can suppress more extensive purine substitutions in the pyrimidine tract than the Tra and Tra2-dependent activity. As negative controls, we replaced the $d s x$ RE with a variety of sequences (data not shown). We found that the antisense of $d s \times R E$ and several other unrelated sequences did not activate splicing. However, we did find that a few se- quences can weakly stimulate splicing. In these cases, the RNA was unstable, and the splicing product was detected only after long exposure of the gel. Given the rather promiscuous RNA-binding property of SR proteins, we believe that these weak stimulatory activities could be ascribed to fortuitous sequence homology to SR protein-binding sites.

Introduction of the purine substitutions in the femalespecific $3^{\prime}$ splice site resulted in a sequence containing equal numbers of purines and pyrimidines and no continuous pyrimidine stretch. However, the mutant $3^{\prime}$ splice site was still activated by the constitutive splicing enhancers. This observation led us to test the possibility that the $d s x$ RE may completely substitute for the function of pyrimidine tract. Thus, we introduced additional purines into the pyrimidine tract. [UCUCUCGAUCUGAUCUAAACCAG/G (wild type) was changed to UCgaUgGAUggGAUggcAAaCAG/G]. These substitutions rendered the sequence preceding the $3^{\prime}$ AG purinerich, and they completely inhibited the constitutive activation of the female-specific 3 ' splice site by the $d s \times R E$ (Fig. 2A, cf. lanes 9 with 7 and 8). Thus, although the constitutive activity of the $d s x$ RE can suppress severe mutations in the pyrimidine tract, it cannot substitute completely for the pyrimidine tract.

\section{Suppression of $5^{\prime}$ splice site mutations by the dsxRE}

To determine whether the $d s x \mathrm{RE}$ can also suppress $5^{\prime}$ splice site mutations, we mutated the $5^{\prime}$ splice site of the $d s x$ minigene transcript. To eliminate or minimize the effect of the $d s \times R E$ on the weak female-specific $3^{\prime}$ splice site, we substituted the original nonconsensus pyrimidine tract (UCUCUCGAUCUGAUCUAAACCAG/G) with the sequence UCUCUCGAUCUUCUCUCCAG/ G. This substitution increases the pyrimidine content of the $3^{\prime}$ splice site and results in constitutive splicing of the intron in the absence of the dsxRE (Fig. 3A, D17, lane 2 ; cf. with D16, lane 1). The original $5^{\prime}$ splice site matches the consensus at eight of nine positions [5' splice site consensus, (C/A)AG/GU(A/G)AGU; $d s x 5^{\prime}$ splice site, AGG/GUAAGU]. In the absence of the $d s \times \mathrm{RE}$, one point mutation in the $5^{\prime}$ splice site (AGc/ GUAAGU; the mutation is the lowercase letter) significantly decreased the level of in vitro splicing activity (Fig. 3A, D18, lane 3). Moreover, no splicing was observed when two point mutations were introduced into the 5' splice site (AGc/GUAAaU; Fig. 3A, D19, lane 4).

When the $d s x$ RE was placed near the $3^{\prime}$ splice site of the intron containing $5^{\prime}$ splice site mutations, it not only stimulated the splicing of the intron containing a single point mutation (Fig. 3B, D20, lane 1) but also led to detectable splicing of the intron with two point mutations in the $5^{\prime}$ splice site (Fig. 3B, D21, lane 2). One complication in comparing the splicing of $5^{\prime}$ splice site mutants with or without the $d s x R E$ is the different stability of the RNAs. In the absence of the $d s x R E$, the RNAs were very unstable. However, even after long exposures of the gel, we could not observe splicing products with D19. Thus, we believe that the splicing product observed with D21 
A

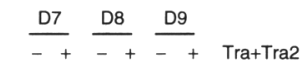
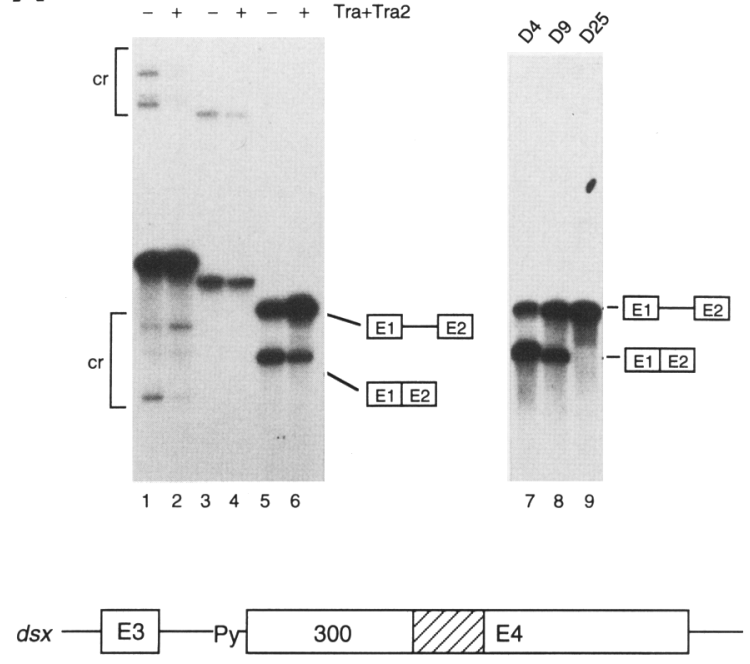

D7

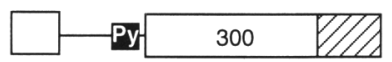

D8

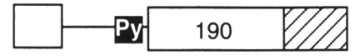

D9

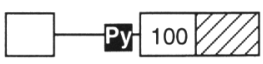

D25

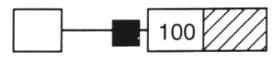

C

$$
0^{3} 0^{2 x} 0^{x}
$$

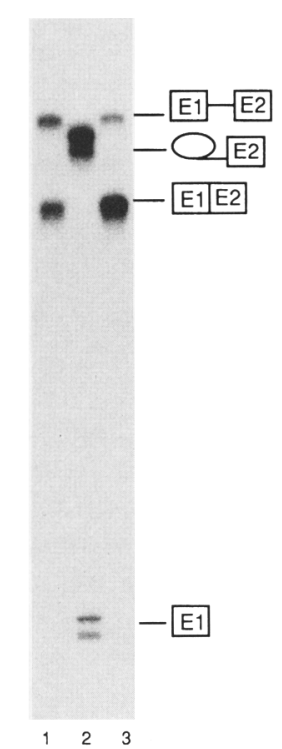

D4

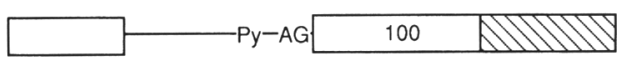

D13

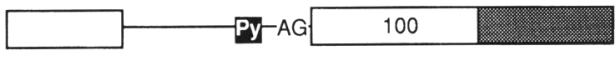

D14

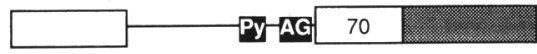

D15

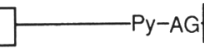

B

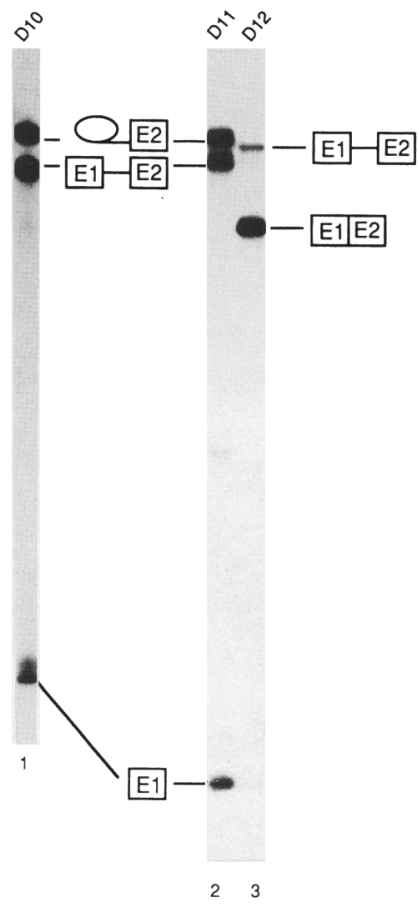

D4

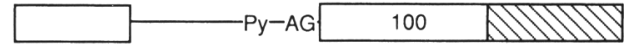

D10

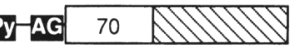

D11

Dy-AG 70

D12

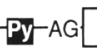

100

Figure 2. Functional interaction between the pyrimidine tract and the dsxRE. (A) Analysis of the effects of purine substitutions in the pyrimidine tract of the female-specific $3^{\prime}$ splice site on in vitro splicing. Py represents the pyrimidine tract in the female-specific 3' splice site. The shaded Py in D7-D9 represents a mutant pyrimidine tract in which three pyrimidines are replaced by purines (see text). The solid box in D25 represents the pyrimidine tract substituted further with purines (see text). (B) Analysis of the effect of pyrimidine tract mutations in the absence of $3^{\prime} \mathrm{AG}$. The accumulated lariat intermediate and the excised $5^{\prime}$ exon are diagramed together with unspliced pre-mRNA and ligated exons. The shaded AG indicates that the $3^{\prime}$ AG of the female-specific $3^{\prime}$ splice site has been deleted. The stippled region in D11 and D12 represents four copies of synthetic repeat sequence. (C) Analysis of the suppression of $3^{\prime}$ splice site mutations by the purine-rich sequence. The shaded area in D13-D15 represents the purine-rich sequence. 
A

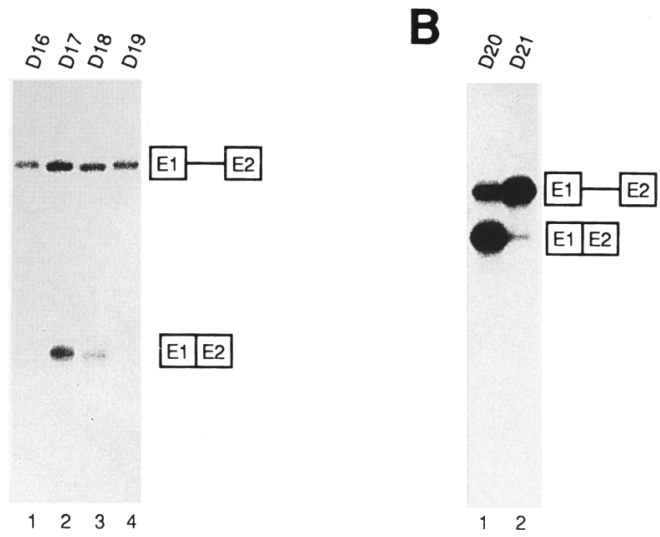

D16

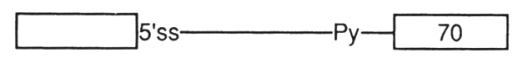

D17

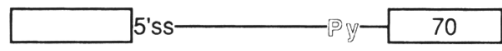

D18

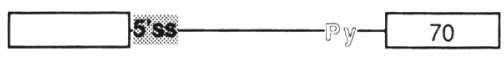

D19

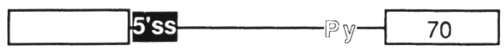

D20

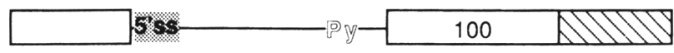

D21

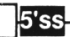

100

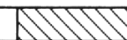

D24

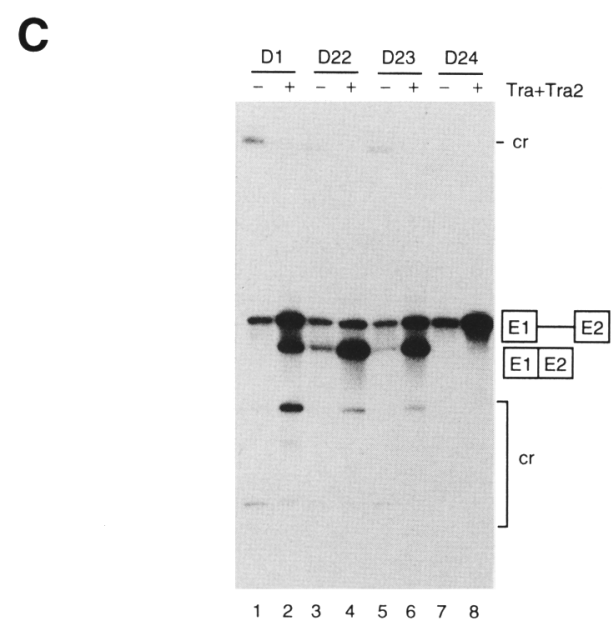

D1

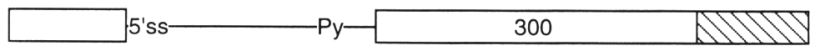

D22

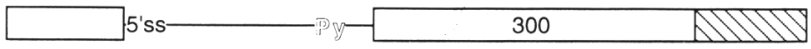

D23

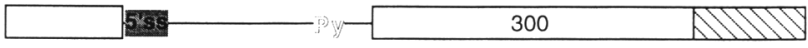

Figure 3. Suppression of 5' splice site mutations by the $d s x$ RE. $(A)$ Analysis of the effects of 5' splice site mutations on pre-mRNAs lacking the $d s x R E$. The outlined Py represents a pyrimidine tract in which the purines have been replaced with pyrimidines. The lightly and darkly shaded 5'SSs represent 5 ' splice sites containing one and two point mutations, respectively. $(B)$ Analysis of the effect of the $d s x R E$ on $5^{\prime}$ splice site mutations. In these experiments the repeat is located only 100 nucleotides from the $3^{\prime}$ splice site and therefore functions in the absence of Tra and Tra2. $(C)$ Analysis of the effect of the dsxRE on $5^{\prime}$ splice site mutations. In these experiments the repeat is located in its normal position and is therefore regulated by Tra and Tra2.

was attributable to splicing activation, rather than an increase in RNA stability. We also tested whether Tra and Tra2 can activate the mutated 5' splice site when the $d s x R E$ is in its original position, 300 nucleotides downstream of the female-specific $3^{\prime}$ splice site (Fig. 3C). Because of the increase in pyrimidine content in the female-specific 3' splice site, constitutive splicing was observed for the pre-mRNAs with either a wild-type 5' splice site or a $5^{\prime}$ splice site containing one point mutation (Fig. 3C, D22 and D23, lanes 3 and 5; cf. D1, lane 1). Tra and Tra 2 could further stimulate the splicing of both pre-mRNAs (Fig. 3C, D22 and D23, lanes 4 and 6; cf. with lanes 3 and 5 , respectively). No splicing was observed when the $5^{\prime}$ splice site contained two point mutations, and the addition of Tra and Tra2 resulted in barely detectable splicing (Fig. 3C, D24, lanes 7 and 8). These results show that both the regulated and constitutive activities of the $d s \times \mathrm{RE}$ can suppress mutations in the $5^{\prime}$ splice site. This suppression could be a direct effect on the $5^{\prime}$ splice site or an indirect consequence of enhancing the strength of the $3^{\prime}$ splice site. The failure of Tra and Tra2 to suppress the more severe $5^{\prime}$ splice site mutation could simply be attributable to the fact that the Tra and Tra-2-dependent activity at a distance is weaker than the constitutive activity of the $d s x \mathrm{RE}$.

To determine whether the $d s x$ RE must be positioned near the $5^{\prime}$ splice site for efficient suppression of $5^{\prime}$ splice site mutations, we placed the $d s x \mathrm{RE}$ in the $5^{\prime}$ exon upstream of the mutated $5^{\prime}$ splice site. We found that the $d s \times R E$ failed to activate the $5^{\prime}$ splice site at this position (data not shown). The suppression of $5^{\prime}$ splice site mutations by the $d s x$ RE was significantly less than that observed with $3^{\prime}$ splice site mutations, suggesting that the $d s x$ RE may act primarily on the $3^{\prime}$ splice site.

Association of SR proteins with the $\mathrm{dsx} R E$ in the presence and absence of Tra and Tra2

We showed previously that Tra and Tra2 promote the assembly of a multicomponent complex (the $d s x$ repeat 
compon the $d s x \mathrm{RE}$, and this complex contains a subset. $5 R$ proteins in addition to Tra and Tra2 (Tian and Maniatis 1993). In this study, we found that the $d s x R E$ can mediate positive regulation in the absence of Tra and Tra2 when placed close to the $3^{\prime}$ splice site. This observation suggests that general splicing factors may associate with the $d s x R E$ in the absence of Tra and Tra2. The association of general splicing factors with the $d s x R E$ in the absence of Tra and Tra 2 may be weak and may therefore have eluded detection in the previous study (Tian and Maniatis 1993). To test this possibility, we scaled up the affinity purification of the repeat complex (Tian and Maniatis 1993) and used the monoclonal antibody mAb 104 (Roth et al. 1991) to probe Western blots of the associated proteins. mAb 104 recognizes all of the known SR proteins through a common phosphoepitope (Zahler et al. 1992). As shown in Figure 4, low levels of SRp30 could indeed be detected in the repeat complex formed in the absence of Tra and Tra2 (lane 2). The association of SRp30 with the repeat complex was specific, because it was not detected in the control complex formed on an RNA without the repeat sequence (Fig. 4, cf. lanes 1 with 2). Consistent with our previous report, Tra and Tra2 significantly increased the amount of SR proteins that stably associate with the complex (Fig. 4, cf. lanes 2 with 3). The prominent band comigrating with SRp40 is most likely attributable to the nonspecific staining of glutathionine $S$-transferase (GST)-R17 protein used in the affinity purification of the complex. The specific association of SR proteins with the $d s x \mathrm{RE}$ in the absence of Tra and Tra 2 is also consistent with observations made in previous UV cross-linking experiments (Tian and Mani-

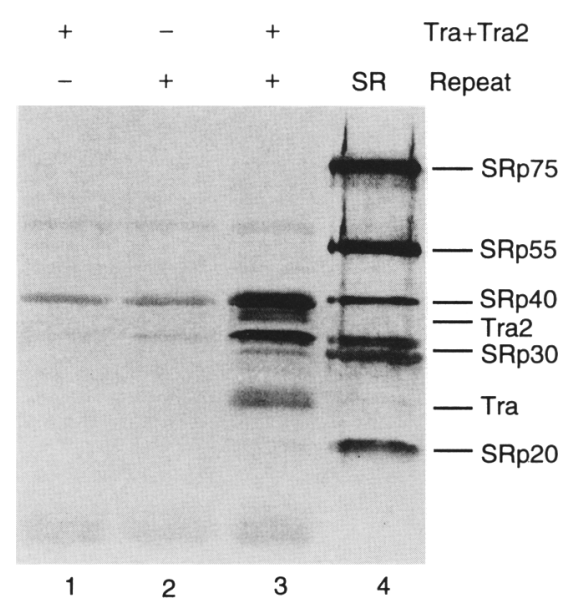

Figure 4. Tra and Tra2-independent association of SR proteins with the $d s x$ RE. The affinity-purified $d s x$ repeat complex was fractionated on an SDS-polyacrylamide gel, the proteins transferred to nitrocellulose, and the filter probed with a monoclonal antibody (mAb 104) that recognizes all of the SR proteins, Tra, and Tra2. Lanes 1-3 contain proteins from complexes formed on RNAs with or without the $d s x$ RE or in the absence or presence of Tra and Tra2, as indicated (top). Lane 4 contains purified SR proteins as positive control. The identities of the bands are indicated to the right of the blot. atis 1992). In the absence of Tra and Tra2, two proteins from nuclear extracts ( 33 and $25 \mathrm{kD}$, respectively) could be specifically cross-linked to the $d s x \mathrm{RE}$. These two proteins most likely correspond to SRp30 and SRp20, because the two cross-linking activities copurify with the SR proteins during the fractionation of nuclear extracts (data not shown). The failure to detect SRp20 in the Western analysis may be attributable to the small amount of this protein in the complex in the absence of Tra and Tra2. SRp30 is a mixture of two SR proteins, SF2/ASF and SC35 (Ge et al. 1991; Krainer et al. 1991; Fu and Maniatis 1992; Zahler et al. 1992). At present, we do not know whether one or both of these proteins are required for the constitutive activity of the $d s \times \mathrm{RE}$.

\section{Discussion}

In previous studies, the $d s x \mathrm{RE}$ was shown to function as a splicing enhancer in the presence of Tra and Tra2, and to exhibit no activity in the absence of these splicing regulatory proteins. In contrast, the purine-rich elements were known to activate splicing constitutively. For this reason, the $d s \times \mathrm{RE}$ was considered distinct from the purine-rich elements. However, in this study we show that the $d s \times R E$ can also act as a constitutive splicing enhancer indistinguishable from the purine-rich elements. When placed within 150 nucleotides of the $3^{\prime}$ splice site, the $d s x$ RE can activate splicing in a Tra and Tra2-independent manner. When separated from the 3 ' splice site by 190-500 nucleotides, the $d s \times R E$ requires Tra and Tra 2 for its activity. Thus, the $d s x$ RE exhibits both constitutive and regulated activities, depending on its distance from the $3^{\prime}$ splice site.

We compared the effect of the constitutive and Tra and Tra2-dependent activities of the $d s x$ RE on mutations in both $3^{\prime}$ and $5^{\prime}$ splice sites. We found that the constitutive activity of the $d s x \mathrm{RE}$ could suppress more extensive purine substitutions in the pyrimidine tract than the Tra and Tra2-dependent regulation. Whether this difference is attributable to the relative strengths of the two enhancer activities or reflects a difference in mechanism remains to be determined.

We also showed that both the constitutive and the Tra and Tra2-dependent activities of the $d s x$ RE could suppress mutations in the $5^{\prime}$ splice site. However, the efficiency of suppression of $5^{\prime}$ splice sites was significantly less than that observed with $3^{\prime}$ splice site mutations. Recently, the purine-rich element of bovine growth hormone pre-mRNA was shown to activate the splicing of an upstream intron containing a weak $5^{\prime}$ splice site (Dirksen et al. 1994). In both of these cases, it remains to be determined whether the enhancers act directly on the weak $5^{\prime}$ splice site or indirectly by increasing the strength of the $3^{\prime}$ splice site. Similarly, we demonstrated previously that the Tra and Tra2-dependent activity of the $d s x$ RE could suppress the effects of mutations in the branchpoint sequence (Tian and Maniatis 1992). Again, this effect could be an indirect consequence of strengthening interactions at the pyrimidine tract rather than 
directly promoting the interactions of splicing components such as U2 snRNP with the branchpoint sequence.

The striking similarity in the ability of the constitutive activity of the $d s x \mathrm{RE}$ and the purine-rich enhancer to suppress purine substitutions in the pyrimidine tract suggests that the two elements may function through a common mechanism. We showed previously that Tra and Tra2 promote the formation of a multiprotein complex on the $d s x R E$, and this complex contains SR proteins and other unidentified proteins (Tian and Maniatis 1993). In this study we show that SR proteins can specifically associate with the $d s x R E$ in the absence of Tra and Tra2, but the level of SR proteins bound to the $d s x R E$ is substantially increased by the addition of $\mathrm{Tra}$ and Tra2. SR proteins have also been shown to bind to purine-rich elements (Lavigueur et al. 1993; Sun et al. 1993b|.

Tra, Tra2, and SR proteins may interact with splicing factors that recognize the pyrimidine tract and thereby stabilize their association with the pyrimidine tract. Two splicing factors have been shown to bind specifically to the pyrimidine tract, U2AF (Zamore and Green 1989, 1991; Zamore et al. 1992) and pyrimidine tractbinding protein (PTB)-associated splicing factor (PSF) (Patton et al. 1993). The splicing factor U2AF is present in the earliest prespliceosome complex E (Bennett et al. 1992) and is required for the formation of the first ATPdependent prespliceosome complex A (Zamore and Green 1989, 1991; Zamore et al. 1992). In contrast, the splicing factor PSF specifically associates with C complex and is essential for the second catalytic step in the splicing reaction (Gozoni et al. 1994). The intron-binding protein (IBP) also binds to the pyrimidine tract, but if it is a functional splicing component it will not act at the earliest steps of spliceosome assembly, because it is specifically associated with U5 snRNA (Gerke and Steitz 1986; Tazi et al. 1986). Finally, a number of heterogeneous nuclear RNP ( $\mathrm{hnRNP}$ ) proteins have been shown to interact with pyrimidine-rich sequences (Swanson and Dreyfuss 1988; Gil et al. 1991; Patton et al. 1991), but these proteins dissociate from pre-mRNA during spliceosome assembly (Staknis and Reed 1994).

Thus, the most likely target for splicing enhancers is U2AF, which consists of two subunits, $\mathrm{U}_{2} \mathrm{AF}^{65}$ and U2AF ${ }^{35}$ (Zamore and Green 1989, 1991; Zamore et al. 1992). This possibility is consistent with a number of observations. First, the purine-rich enhancer has been shown to promote A complex formation (Sun et al. 1993a) and could also be required for E complex assembly. Second, U2AF is the only known pyrimidine tractbinding protein that has been detected in $\mathrm{E}$ complex (Bennett et al. 1992). Third, Tra, Tra2, and SR proteins can interact with one another through the RS domain (Wu and Maniatis 1993; Amrein et al. 1994), and each of these proteins can interact, in turn, with $\mathrm{U}_{2} \mathrm{AF}^{35} \mathrm{Wu}$ and Maniatis 1993). Thus, the SR proteins bound to the splicing enhancers may interact with $\mathrm{U}_{2} \mathrm{AF}^{35}$ to stabilize the binding of the U2AF heterodimer to the pyrimidine tract.

The interaction between the SR proteins and U2AF may also provide an explanation for the observation that when it is close to the $3^{\prime}$ splice site, the activity of $d s \times R E$ is independent of Tra and Tra 2 and can suppress more extensive purine substitutions in the pyrimidine tract. According to the model of Figure 5, the intrinsic affinity of U2AF for the weak 3 ' splice site and that of the SR proteins for the $d s \times R E$ is low (Fig. 5A). The close proximity of the $d s \times R E$ and the $3^{\prime}$ splice site may facilitate cooperative interactions between U2AF and the SR proteins (Fig. 5B). This cooperative interaction would mutually stabilize their individually weak binding to their respective binding sites. In contrast, when the $d s \times R E$ is at a distance from the $3^{\prime}$ splice site, this cooperative interaction would not occur in the absence of Tra and Tra2 (Fig. 5C). Thus, the SR proteins and U2AF would dissociate from the RNA before they could interact. However, according to the model, Tra and Tra2 would
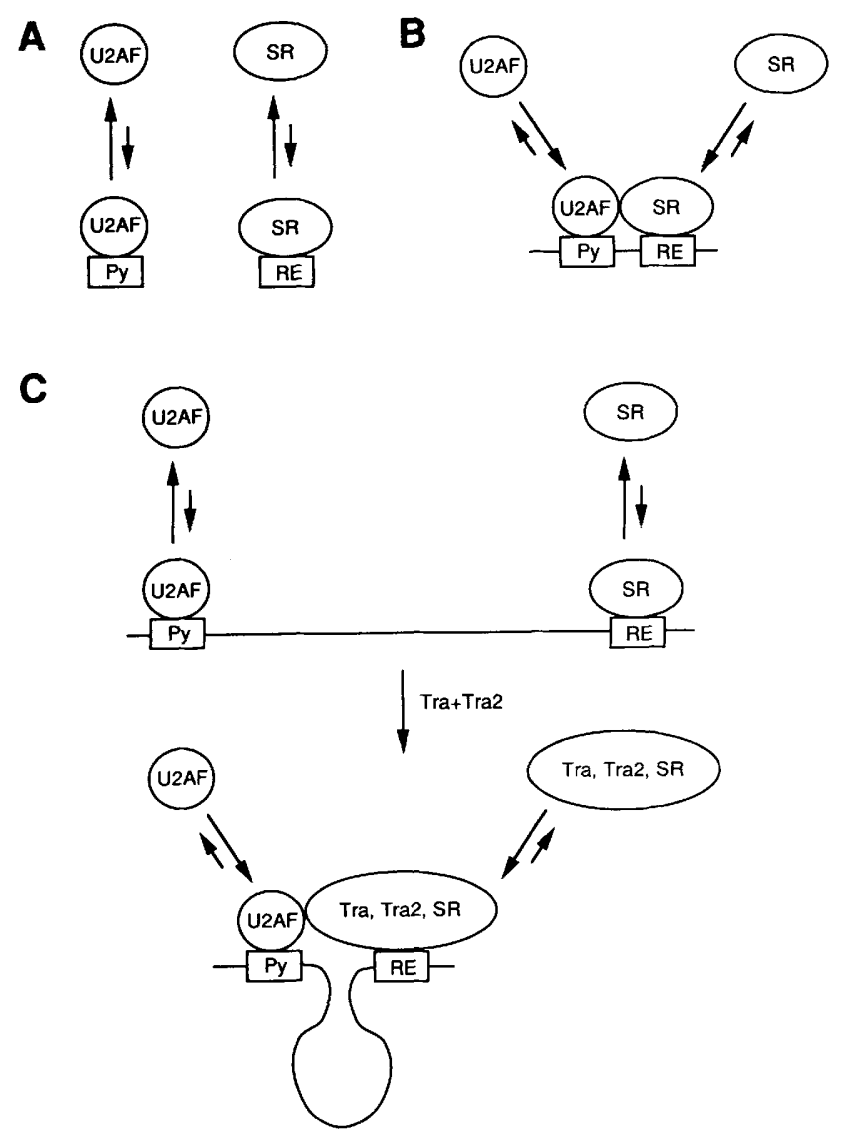

Figure 5. Model for the mechanism of the constitutive and the Tra and Tra2-dependent activities of the $d s x$ RE. (A) Individually, U2AF and SR proteins bind weakly to a nonconsensus pyrimidine tract $(\mathrm{Py})$ and the $d s x \mathrm{RE}(\mathrm{RE})$, respectively. $(B) \mathrm{Co}-$ operative interactions between SR proteins and U2AF stabilize their association with the $d s \times \mathrm{RE}$ (RE) and the pyrimidine tract (Py), respectively, when the two binding sites are in close proximity. (C) Tra and Tra2 stabilize the binding of SR proteins to the $d s x \mathrm{RE}(\mathrm{RE})$ and facilitate the cooperative interactions between the splicing enhancer complex with U2AF at the distant 3 ' splice site. 
stabilize the association of SR proteins with the $d s \times R E$, thus facilitating cooperative interactions between the splicing enhancer complex and U2AF.

\section{Materials and methods \\ In vitro splicing reactions}

In vitro splicing reactions were carried out as described previously (Tian and Maniatis 1992).

\section{In vitro splicing substrates}

D1 contains 84 nucleotides of $d s x$ exon 3, the complete intron between exon 3 and 4 (114 nucleotides), and 536 nucleotides of exon 4. D2, D3, and D4 contain progressively larger deletions starting 30 nucleotides from the first copy of the repeat sequence. The number of nucleotides between the repeat sequence and the female-specific 3 ' splice site after the deletion is indicated in the figures. D7, D8, and D9 are derived from D1, $\mathrm{D} 2$, and $\mathrm{D} 4$, respectively, with mutations in the pyrimidine tract of the female-specific $3^{\prime}$ splice site (TCTCTCGATCTGATCTAAACCAG/G is mutated to TCgaTgGATCTGATCTAAACCAG/G). D10 is derived from D9 by deleting a 30 nucleotide fragment containing the $3^{\prime}$ AG. D 11 and D12 are derived from D10 and D9, respectively, by replacing the $d s x$ repeat region with four copies of an oligonucleotide corresponding to the second copy of the repeat sequence (GGATCCGTCTTCAATCAACATACGCGAGATCC; the repeat sequence is underlined). D13, D14, and D15 are derived from D9, D10, and D4, respectively, by replacing the repeat region with three copies of an oligonucleotide corresponding to the S3 element in the IgM M2 exon (CATGGAAGGACAGCACAG; the S3 element is underlined). D16 contains 84 nucleotides of exon 3 , the intron between exon 3 and 4 (114 nucleotides), and 70 nucleotides of exon 4 . Dl7 is derived from Dl6 with pyrimidine substitutions in the pyrimidine tract of the female-specific $3^{\prime}$ splice site (TCTCTCGATCTGATCTAAACCAG/G is mutated to TCTCTCGATCTTCTCTCCAG/G). D18 and D19 are derived from D17 with mutations in the 5 ' splice site [AGG/GUAAGU is mutated to AGc/GUAAGU (D18), or AGc/GUAAaU (D19)]. D20 and D2 1 are derived from D18 and D19 with the addition of the repeat region. D22 is derived from D1 with pyrimidine substitutions in the pyrimidine tract as in D17. D23 and D24 are derived from D22 with $5^{\prime}$ splice site mutations as in D18 and D19, respectively. D25 is derived from D9, with additional purine substitutions in the pyrimidine tract [TCGATGGATCTGATCTAAACCAG/G (D9) is mutated to TCGATGGATggGATggcAAaCAG/G (D25); the mutations are in lowercase letters]. $\mathrm{D} 26$ is derived from $\mathrm{D} 1$ by replacing the repeat region with three copies of the S3 purine-rich sequence. D27 is derived from Dl by duplicating a 200 -bp fragment between the female-specific 3 ' splice site and the repeat region.

\section{Acknowledgments}

We thank Drs. R. Reed, M. Bennett, and members of the Maniatis group for discussion and critical comments on the manuscript. We are grateful to Dr. M. Roth for providing the hybridoma producing mAb 104. M. Tian is supported by a Merck predoctoral fellowship. This work is funded by a grant from the National Institutes of Health to T.M.

The publication costs of this article were defrayed in part by payment of page charges. This article must therefore be hereby marked "advertisement" in accordance with 18 USC section 1734 solely to indicate this fact.

\section{References}

Amrein, H., M. Gorman, and R. Nöthiger. 1988. The sex-determining gene tra-2 of Drosophila encodes a putative RNA binding protein. Cell 55: 1025-1035.

Amrein, H., M. Hedley, and T. Maniatis. 1994. The role of specific protein-RNA and protein-protein interactions in positive and negative control of pre-mRNA splicing by transformer 2. Cell 76: 735-746.

Bandziulis, R.J., S.M. Swanson, and G. Dreyfuss. 1989. RNAbinding proteins as developmental regulators. Genes \& Dev. 3: $431-437$.

Bennett, M., S. Michaud, J. Kingston, and R. Reed. 1992. Protein components specifically associated with pre-spliceosome and spliceosome complexes. Genes \& Dev. 6: 1986-2000.

Boggs, R.T., P. Gregor, S. Idriss, J.M. Belote, and M. McKeown. 1987. Regulation of sexual differentiation in D. melanogaster via alternative splicing of RNA from the transformer gene. Cell 50: 739-747.

Burtis, K.C. and B.S. Baker. 1989. Drosophila doublesex gene controls somatic sexual differentiation by producing alternative spliced mRNAs encoding related sex-specific polypeptides. Cell 56: 997-1010.

Chou, T.-B., Z. Zachar, and P.M. Bingham. 1987. Developmental expression of a regulatory gene is programmed at the level of splicing. EMBO /. 6: 4095-4104.

Dirksen, W.P., R.K. Hampson, Q. Sun, and F.M. Rottman. 1994. A purine-rich exon sequence enhances alternative splicing of bovine growth hormone pre-mRNA. I. Biol. Chem. 269: 6431-6436.

Fu, X.-D. and T. Maniatis. 1990. Factor required for mammalian spliceosome assembly is localized to discrete regions in the nucleus. Nature 343: 437-441.

- 1992. Isolation of a complementary DNA that encodes the mammalian splicing factor SC35. Science 256: 535-538.

Ge, H., P. Zuo, and J.L. Manley. 1991. Primary structure of the human splicing factor ASF reveals similarities with Drosophila regulators. Cell 66: 373-382.

Gerke, V. and J. Steitz. 1986. A protein associated with small nuclear ribonucleoprotein particles recognizes the 3 ' splice site of pre-messenger RNA. Cell 47: 973-984.

Gil, A., P.A. Sharp, S.F. Jamison, and M.A. Garcia-Blanco. 1991. Characterization of cDNAs encoding the polypyrimidine tract-binding protein. Genes \& Dev. 5: 1224-1236.

Goralski, T.J., J.-E. Edstrom, and B.S. Baker. 1989. The sex determination locus transformer-2 of Drosophila encodes a polypeptide with similarity to RNA binding proteins. Cell 56: 1011-1018.

Gozoni, O., J.G. Patton, and R. Reed. 1994. A novel set of spliceosome associated proteins (SAPs) and the essential splicing factor PSF bind stably to pre-mRNA prior to catalytic step 2 of the splicing reaction. EMBO $/$. (in press).

Hedley, M. and T. Maniatis. 1991. Sex-specific splicing and polyadenylation of $d s x$ pre-mRNA requires a sequence that binds specifically to tra-2 proteins in vitro. Cell 65: 579-586.

Hoshijima, K., K. Inoue, I. Higushi, H. Sakamoto, and Y. Shimura. 1991. Control of doublesex alternative splicing by transformer and transformer-2 in Drosophila. Science 252: 833-836.

Kenan, D.J., C.C. Query, and J.D. Keene. 1991. RNA recognition: Towards identifying determinants of specificity. Trends Biochem. Sci. 16: 214-220. 
Kohtz, J.D., S.F. Jamison, C.L. Will, P. Zuo, R. Lührmann, M.A. Garcia-Blanco, and J.L. Manley. 1994. Protein-protein interactions and $5^{\prime}$ splice site recognition in mammalian mRNA precursors. Nature 368: 119-124.

Krainer, A.R., A. Mayeda, D. Kozak, and G. Binns. 1991. Functional expression of clones of human splicing factor SF2: Homology to RNA binding proteins, Ul 70K, and Drosophila splicing regulators. Cell 66: 383-394.

Lavigueur, H. L. Branche, A.R. Kornblihtt, and B. Chabot. 1993. A splicing enhancer in the human fibronectin alternate EDl exon interacts with SR proteins and stimulates U2 snRNP binding. Genes \& Dev. 7: 2405-2417.

Li, H. and P.M. Bingham. 1991. Arginine/serine-rich domains of the $s u\left(w^{a}\right)$ and tra RNA processing regulators target proteins to a subnuclear compartment implicated in splicing. Cell 67: 335-342.

Mancebo, R., P.C.H. Lo, and S.M. Mount. 1990. Structure and expression of the Drosophila melanogaster gene for the $\mathrm{U} 1$ small nuclear ribonucleoprotein particle $70 \mathrm{~K}$ protein. Mol. Cell. Biol. 10: 2492-2502.

Nagoshi, R.N. and B.S. Baker. 1990. Regulation of sex-specific RNA splicing at the Drosophila doublesex gene: Cis-acting mutations in exon sequences alter sex-specific RNA splicing patterns. Genes \& Dev. 4: 89-97.

Patton, J.G., S.A. Mayer, P. Tempst, and B. Nadal-Ginard. 1991. Characterization and molecular cloning of polypyrimidine tract-binding protein: A component of a complex necessary for pre-mRNA splicing. Genes \& Dev. 5: 1237-1251.

Patton, J.G., E.B. Porro, J. Galceran, P. Tempst, and B. NadalGinard. 1993. Cloning and characterization of PSF, a noval pre-mRNA splicing factor. Genes \& Dev. 7: 393-406.

Reed, R. 1989. The organization of $3^{\prime}$ splice-site sequences in mammalian introns. Genes \& Dev. 3: 2113-2123.

Roth, M.B., A.M. Zahler, and J.A. Stolk. 1991. A conserved family of nuclear phosphoproteins localized to sites of polymerase II transcription. J. Cell Biol. 115: 587-596.

Ryner, L.C. and B.S. Baker. 1991. Regulation of doublesex premRNA processing occurs by 3 '-splice site activation. Genes \& Dev. 5: 2071-2085.

Spector, D.L., X.-D. Fu, and T. Maniatis. 1991. Associations between distinct pre-mRNA splicing components and the cell nucleus. EMBO $/$. 10: $3467-3481$.

Staknis, D. and R. Reed. 1994. Direct interactions between premRNA and six U2 small nuclear ribonucleoproteins during spliceosome assembly. Mol. Cell. Biol. 14: 2994-3005.

Sun, Q., R.K. Hampson, and F.M. Rottman. 1993a. In vitro analysis of bovine growth hormone pre-mRNA alternative splicing. J. Biol. Chem. 268: 15659-15666.

Sun, Q., A. Mayeda, R.K. Hampson, A.R. Krainer, and F.M. Rottman. 1993b. General splicing factor SF2/ASF promotes alternative splicing by binding to an exonic splicing enhancer. Genes \& Dev. 7: 2598-2608.

Swanson, M. and G. Dreyfuss. 1988. RNA binding specificity of hnRNP proteins: A subset bind to the $3^{\prime}$ end of introns. EMBO I. 7: 3519-3529.

Tanaka, K., A. Watakabe, and Y. Shimura. 1994. Polypurine sequences within a downstream exon function as a splicing enhancer. Mol. Cell. Biol. 14: 1347-1354.

Tazi, J., C. Alibert, J. Temsamani, I. Reveillaud, G. Cathala, C. Brunel, and P. Jeanteur. 1986. A protein that specifically recognizes the $3^{\prime}$ splice site of mammalian pre-mRNA introns is associated with a small nuclear ribonucleoprotein. Cell 47: 755-766.

Theissen, H., M. Etzerodt, R. Reuter, C. Schneider, F. Lottspeich, P. Argos, R. Luhrman, and L. Philipson. 1986. Cloning of the human cDNA for the U1 RNA-associated $70 \mathrm{~K}$ protein.
EMBO I. 5: 3209-3217.

Tian, M. and T. Maniatis. 1992. Positive control of pre-mRNA splicing in vitro. Science 256: 237-240.

. 1993. A splicing enhancer complex controls alternative splicing of doublesex pre-mRNA. Cell 74: 105-114.

Watakabe, A., K. Tanaka, and Y. Shimura. 1993. The role of exon sequences in splice site selection. Genes \& Dev. 7: $407-418$.

Wu, J.Y. and T. Maniatis. 1993. Specific interactions between proteins implicated in splice site selection and regulated alternative splicing. Cell 75: 1061-1070.

$\mathrm{Xu}, \mathrm{R} ., \mathrm{J}$. Teng, and T.A. Cooper. 1993. The cardiac troponin T alternative exon contains a novel purine-rich positive splicing element. Mol. Cell. Biol. 13: 3660-3674.

Zahler, A.M., S.W. Lane, J.A. Stolk, and M.B. Roth. 1992. SR proteins: A conserved family of pre-mRNA splicing factors. Genes \& Dev. 6: 837-847.

Zamore, P.D. and M.R. Green. 1989. Purification and biochemical characterization of U2 small nuclear ribonucleoprotein auxiliary factor. Proc. Natl. Acad. Sci. 86: 9243-9247.

- 1991. Biochemical characterization of U2 snRNP auxiliary factor: An essential pre-mRNA splicing factor with a novel intranuclear distribution. EMBO /. 10: 207-214.

Zamore, P.D., J. Patton, and M.R. Green. 1992. Cloning and domain structure of the mammalian splicing factor U2AF. Nature 355: 609-614. 


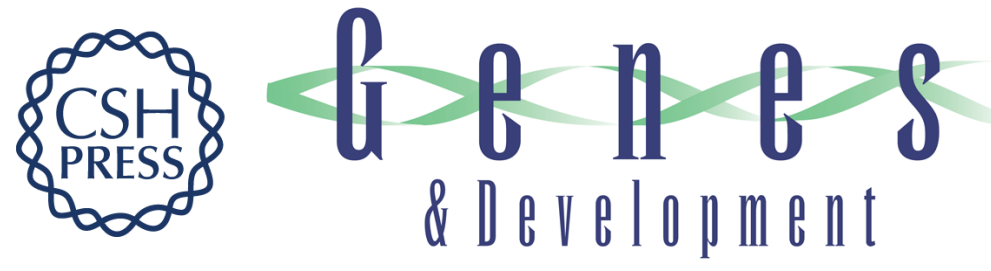

\section{A splicing enhancer exhibits both constitutive and regulated activities.}

M Tian and T Maniatis

Genes Dev. 1994, 8:

Access the most recent version at doi:10.1101/gad.8.14.1703

References This article cites 45 articles, 23 of which can be accessed free at:

http://genesdev.cshlp.org/content/8/14/1703.full.html\#ref-list-1

License

Email Alerting

Service

Receive free email alerts when new articles cite this article - sign up in the box at the top right corner of the article or click here.

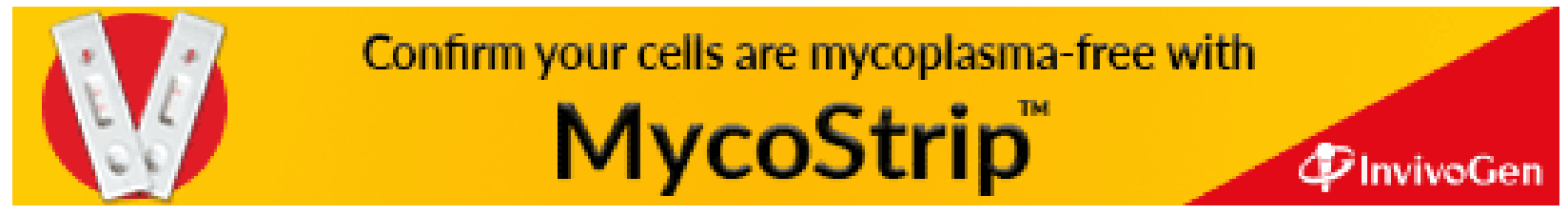

